\title{
Serological and entomological survey of canine leishmaniasis in Lampedusa island, Italy
}

\author{
Valentina Foglia Manzillo ${ }^{1}$, Manuela Gizzarelli ${ }^{1 *}$ (D), Fabrizio Vitale², Serena Montagnaro ${ }^{1}$, Alessandra Torina², \\ Salvo Sotera ${ }^{3}$ and Gaetano Oliva ${ }^{1}$
}

\begin{abstract}
Background: During last decade Lampedusa island (Italy) has been interested by a deep social change caused by the massive arrival of migrants from north Africa. The goal of this study was to evaluate current CanL burden and risk factors for Visceral Leishmaniosis (VL) on Lampedusa, actually based on very few data obtained in a previous study performed fifteen years ago. Two hundred and forty-two dogs were enrolled for the detection of Leishmania infantum infection by serology. In addition, an entomological investigation was performed to confirm the presence of Leishmania-vectors.
\end{abstract}

Results: Seroprevalence was of 54.13\%. 223 sand flies specimens were collected. Among them, 4 species were identified: Phlebotomus perniciosus, P. papatasi, P. neglectus, Sergentomia minuta, with P. perniciosus the most abundant $(67.7 \% ; p<0.01)$.

Conclusion: The high proportion of seropositive dogs together with the presence of the most competent vector for $L$. infantum, P. perniciosus, demonstrate that $L$. infantum abundantly circulates in the island and may constitute a risk for people, particularly for hosted migrants.

Keywords: Epidemiology, Canine leishmaniosis, Sand flies, Zoonosis, Lampedusa island

\section{Background}

Zoonotic Visceral Leishmaniosis (ZVL) is an important zoonotic disease which is associated with the long history of companionship between dogs and humans. ZVL, caused by Leishmania infantum parasites is transmitted by phlebotomine sand flies vectors. Despite provoking a limited number of overt human clinical cases - in comparison with global leishmaniosis figures $-L$. infantum represents a latent public health threat in the European Union (EU) because studies performed in several endemic foci have disclosed a high prevalence of asymptomatic parasite carriers [1]. Based on recent data, Canine Leishmaniosis (CanL) affects approximately 2.5 million dogs in Mediterranean and peri-Mediterranean areas each year [2]. CanL is characterized by different

\footnotetext{
* Correspondence: manuela.gizzarelli@unina.it

${ }^{1}$ Department of Veterinary Medicine and Animal Production, University of Naples Federico II, 80137 Naples, Italy

Full list of author information is available at the end of the article
}

prevalence rates in reason of the geographical climate condition that allow or not the presence and abundance of sand fly vectors. L. infantum causes about 700 autochthonous cases of human VL each year in Mediterranean basin. The average seroprevalence in domestic dogs is less than $25 \%[3,4]$. Sporadic human cases of VL and cutaneous leishmaniasis (CL) caused by L. infantum have been reported from Lampedusa. CanL is known to occur in the island, but studies on infection prevalence are limited to one study performed fifteen years ago [5]. In that trial, a seroprevalence of $39,1 \%$ was found in stray dogs housed in an animal shelter. Three species of sand flies were identified to be present on the island, Phlebotomus papatasi was the most prevalent $(88,8 \%)$. P. perniciosus $(9,7 \%)$ and Sergentomya minuta $(1,5 \%)$ were also found. During the last decade Lampedusa has experienced massive migration from north Africa. These refugers are sheltered temporarily in an emergency center near the one village, before moving to other 
European locations. The dog's population has also changed due to closure of animal shelter and adoption of dogs by tourists visiting the island during the summer. At present, more than $95 \%$ of the dog are owned. Based on these dramatic shifts in both the human and canine population the goal of this study was to evaluate current CanL burden and risk factors for VL on Lampedusa.

\section{Methods}

\section{Study site}

The present study was carried out in Lampedusa, a small island $\left(20,2 \mathrm{~km}^{2}\right)$ in the Italian Pelagie archipelago $\left(35^{\circ}\right.$ $\left.30^{\prime} 56^{\prime \prime} \mathrm{N} \mathrm{12} 2^{\circ} 34^{\prime} 23^{\prime \prime} \mathrm{E}\right)$. This archipelago is the southernmost territory of Italy (205 km from Sicily) and the nearest to North Africa $(113 \mathrm{~km})$. The island has calcareous soil and is characterized by the absence of forest trees, because of intense deforestation at the beginning of the last century. Compared with other places sited at the same latitude, Lampedusa has a mild climate due to consistent winds, that are strong enough to prevent normal development of vegetation. The maximum altitude is $113 \mathrm{~m}$; rivers are not present and water sources are extremely scarce. During the period from April to October temperature can reach up to $30{ }^{\circ} \mathrm{C}$ (www.il meteo.it/ archiviostorico). Lampedusa has a population of 6000 inhabitants; the most of them concentrated in a small village situated in the southeast of the island. Dogs largely represent the most abundant domestic animals present on the island; less than 1000 dogs have been estimated. A few small goat and sheep farms are in the central part of the island.

\section{Study design}

The study was designed to investigate serological, clinical and parasitological findings from autochthonous dogs living on Lampedusa and to study the phlebotomine distribution on the island, to obtain useful information about the possible risk for human population. Two hundred and forty-two dogs were enrolled between mid-November 2016 to early April 2017, a period with no adult sand flies. All dogs were owned. The owners were selected among a larger group participating to a free campaign to get information on CanL prevention, carried out during routinary veterinary clinical activity. Their dogs were enrolled after the signature of an informed consent. Dogs were of different sex, breed and age. The large majority of dogs enrolled in the study were born on the island, all the others were imported there when puppies. Each dog was submitted to clinical examination and blood sample. Clinical assessment was performed by accurate inspection of dogs for the presence of any physical signs attributable to Leishmania infection. All data were recorded on a clinical form that included, signaling, history, particularly including the use repellents, and clinical signs, classified in the following categories: 1) Systemic signs (S), including weight loss, muscular atrophy, lethargy and pale mucous membranes; 2) Reticulo-endothelial signs (RE), including enlargement of palpable lymph nodes such as submandibular, prescapular or popliteal nodes, and splenic enlargement as determined by palpation; 3) Cutaneous signs (C), including nodules/ulcers, onychogryphosis, dry exfoliative dermatitis and alopecia; 4) Ocular signs (O), including blepharitis, keratoconjunctivitis and uveitis [6]. The collection of biological samples was performed in accordance with the national guidelines for animal welfare. Ten milliliters of blood were obtained from the jugular vein and was centrifugated to take serum for serology, then stored $-20{ }^{\circ} \mathrm{C}$. Dogs showing clinical signs suggestive of CanL were submitted to lymph node sampling to perform qPCR useful for therapeutic follow up. Lymph node aspiration was performed from popliteal lymph nodes using fine needle aspiration technique [7]. For this purpose owners signed a different informed consent.

\section{Serology}

The presence of anti-Leishmania antibodies was assessed by an indirect immunofluorescence antibody test (IFAT) in conformity of recommendation of OIE [8] using MHOM/TN/80/IPT1 as a whole parasite antigen preparation fixed on multispot slides (Bio Merieux Spa, Florence, Italy) and a fluorescent labeled anti-canine gamma globulin (Sigma Aldrich, Milan, Italy) as conjugate. Positive sera were serially diluted and tested to establish the maximum reaction titre, starting from dilution at concentration of 1:40. Positive and negative controls were included in each slide. A dog was defined as infected if positive to quantitative serology at cut-off $1: 80$, according to Italian National Reference Centre for Leishmaniasis (C. Re.Na.L - Istituto Zooprofilattico Palermo, Italy).

\section{qPCR}

DNA was extracted from lymph node aspirate samples using a Invitrogen PureLink Genomic DNA Mini Kit according to manufacturer's instructions. The PCR test was targeted to a $68 \mathrm{bp}$ fragment inside the constant region of kinetoplast DNA (kDNA) (NCBI accession number AF291093) as previously described [9]. The primer sequences were: QLK2-U 5'-GGCGTTCTGCGAAAACCG -3'; QLK2-D5'-AAAATGGCATTTTCGGGCC-3'; probe: 5'-TGGGTGCAGAAATCCCGTTCA-3' 5'FAM with 3' BHQ labelled. Each amplification was performed in duplicate, in $20 \mu \mathrm{l}$ reaction mixture containing $1 \times$ TaqMan Universal Master Mix (Applied Biosystem), $20 \mathrm{pmol} / \mu \mathrm{l}$ primers and $10 \mathrm{pmol} / \mu \mathrm{l}$ of probe (Qleish 2), $1 \times$ EXO IPC Mix, $1 \times$ EXO IPC DNA. The thermal cycling conditions were: incubation for $2^{\prime}$ at $50^{\circ} \mathrm{C}$ for uracil-N-glycosylase activity. This step was followed by a $10^{\prime}$ denaturation at $95^{\circ}$ 
$\mathrm{C}$ and 45 cycles at $95^{\circ} \mathrm{C}$ for $15^{\prime \prime}$ and $60^{\circ} \mathrm{C}$ for $1^{\prime}$ each. Results were expressed as a parasite count for $\mathrm{ml}$ calibrated to a standard curve. Standard curve DNA was extracted from cultured IPT1 MON1, obtained from the collection C.Re.Na.L, $1 \times 10^{9}$ cells $/ \mathrm{ml}$ and homogenized in $1 \mathrm{ml}$ of lysis mix, (1\% Tween 20, 1\% Non idet P-40, e 20\% Chelex). Then decimal serial dilutions of the stock solution were performed to obtain the points of the curve ranging from the DNA equivalent of $1 \times 10^{6}$ cells to $1 \mathrm{cell} / \mathrm{ml}$.

\section{Entomological findings}

In addition, a phlebotomine sand flies survey was performed to confirm the presence of Leishmania-competent vectors and to evaluate possible variations in their distribution in urban/periurban and rural areas of the island. Sticky traps and CDC miniature light traps (Hausherr's Machine Works, Toms River, NJ, USA) were used in five urban ( $n=2$ traps), periurban ( $n=1$ trap) and rural ( $\mathrm{n}=2$ traps) sites of the island during 2 weeks of sand flies collection (first week: middle June, second week: middle July 2016). Urban sites consisted of 2 dog's owner private homes that housing two and four dogs respectively: the periurban site was a house with courtyard housing six dogs. The rural sites consisted of two small farms where goats and sheep were bred. Sticky traps were positioned in the same locations. CDC traps (one at each site) were suspended at approximately $1.5 \mathrm{~m}$ above the ground and were operated from $1 \mathrm{~h}$ before sunset until $1 \mathrm{~h}$ after sunrise. The light traps were retrieved each morning and the collected phlebotomines stored in alcohol prior to identification following standard taxonomic keys. Molecular method was used for the confirmation and differentiation of sand fly species including polymerase chain reaction (PCR) of the ribosomal ITS2 region PCR as PCR-RFLP were used for genome and species confirmation [10,11]. As there is not a clear urban vs. periurban area, these areas as entomological results were combined.

\section{Statistical methods}

Sample size was determined [12] with the following information: expected canine prevalence of $L$. infantum (30\%) based on the average prevalence of high endemic area for SOUTH ITALY [13], confidence interval (95\%) and desired absolute precision (7\%). Leishmania seroprevalence in dogs, according to the various characteristics (gender, age, and the use of ectoparasiticides) was calculated with an associated 95\% confidence interval (CI). The apparent prevalence of $L$. infantum infection was calculated as the number of serologically positive dogs among the total number of dogs tested.

The true prevalence TP was estimated using standard methods [12]:

$$
\mathrm{TP}=(\mathrm{AP}+\mathrm{Sp}-1) /(\mathrm{Se}+\mathrm{Sp}-1)
$$

Where

$\mathrm{AP}$ is the apparent prevalence;

Se is the sensitivity of the test;

Sp is the specificity of the test.

IFAT test has a sensitivity of $96 \%$ and specificity of $98 \%$ [8].

Differences in prevalence between these various groups were assessed by the two-sided chi-square $t$ test. A $P$ value $<0.05$ was considered statistically significant.

The variables were then applied to binary logistic models to find Odds ratios for factors associated with the seroprevalence.

The sand flies species distribution according to gender and living area was calculated with an associated 95\% confidence interval (CI).

The statistical analyses were performed using MedCalc software (Frank Shoonjans, V.7.2.1.0) and SPSS, version 13.0 for Windows.

\section{Results}

A total of $131 / 242$ samples $(54,13 \%)$ resulted positive to IFAT. Twenty-three seropositive dogs (13.4\%) had clinical signs; 19 resulted positive on q-PCR. Based on the classification of clinical signs, the most frequent were RE (52.6\%), particularly lymph node enlargement, S (42.1\%), as weight loss and C (36.8\%), mainly dry exfoliative dermatitis. Statistical analysis was performed only in dogs for which complete anamnestic data were recorded (n. 176; positive dogs n. 116). No difference between positive and negative dogs was assessed in reason of the age and the use of ectoparasiticides (Table 1). 223 sand flies specimens were collected; 214 have been used for species identification. Among them, 4 species were identified: $P$. perniciosus, $P$. papatasi, $P$. neglectus, S. minuta. P. perniciosus was the most abundant $(67.7 \% ; p<0.01)$, followed by $S$. minuta $(28.5 \%)$ and $P$. papatasi $(3.3 \%)$. Only one sand fly was identified as $P$. neglectus $(0,5 \%)$. Phlebotomines were most present in rural area $(71.96 \% ; p<0.0001)$, where female insects resulted prevalent $(76 \% ; p<0.05)$. Sand flies distribution are summarized in Table 2.

\section{Discussion}

The main risk factors for ZVL in people is CanL prevalence in that area. CanL represents a severe problem among dogs of South Italy, the median 30 years prevalence calculated from 377 canine serosurveys performed in Italy dogs was $18 \%$ (range of 11-21\%) [4] . More than $1 / 3$ of Italian municipalities were endemic for CanL [14]. On the island of Lampedusa more than $50 \%$ of dogs tested were seropositive. In this community, most dogs share the same circumscribed environment as their owners, and live in urban/periurban areas. CanL in 
Table 1 Influence of age and ectoparasiticide use on Leishmania positive dogs

\begin{tabular}{|c|c|c|c|c|c|c|c|c|}
\hline Factor & $n$ & $\%$ & Standard Error \% & $95 \% \mathrm{Cl}$ & Chi-square & $P$ & OR & $95 \% \mathrm{Cl}$ \\
\hline Total & 116 & 65.90 & \pm 7.00 & $58.9-72.9$ & - & - & - & - \\
\hline \multicolumn{9}{|l|}{ Age } \\
\hline$<5$ & 79 & 44.80 & \pm 7.3 & $37.40-52.13$ & 1.563 & 0.2112 & 0.5906 & $0.2852-1.2228$ \\
\hline$>5$ & 37 & 21.01 & \pm 6.02 & $14.99-27.03$ & & & & \\
\hline \multicolumn{9}{|c|}{ Anti-ectoparasite drugs } \\
\hline yes & 66 & 37.50 & \pm 7.15 & $30.35-44.65$ & 0.017 & 0.8955 & 1.0094 & $0.5381-1.8936$ \\
\hline no & 50 & 28.40 & \pm 6.63 & $21.77-35.03$ & & & & \\
\hline
\end{tabular}

Lampedusa should be very stable, because most dogs are autochthonous and the young/middle age did not result a risk factor for the development of the infection. Most owners of infected dogs declared that they applied insecticide drugs. However, it was not possible to have detailed information about their confidence with the use of these compounds nor regarding the composition of the applied substances. This bias could justify the absence of statistical differences between positive dogs using or not insecticide drugs. The clinical signs detected on positive dogs did not differ from those usually described for CanL, and were all related to chronic form of the disease, characterized by high IFAT titer. In addition, many owners of sick dogs declared that they do not treat with specific anti-Leishmania drugs, or that the dogs after the first treatment were not submitted to a regular follow up. The existence of sick not treated dogs could also influence the high circulation of the parasite on the island. Four different species of sand flies were recorded, two of which are considered competent vectors of $L$. infantum. In contrast to a previous study [5] $P$. papatasi was not abundant in the trapped specimens. The difference could be due to the different way of trapping used in the two studies, the first by using mainly direct mouth aspirators near kennel or inside hen-houses, the second with the use of light traps that are less attractive for this phlebotomine species. The presence of this sand fly on the

Table 2 Phlebotomine sand flies distribution on Lampedusa island

\begin{tabular}{llll}
\hline Area & Species & $n$ & $\%$ \\
\hline Urban/periurban Area & Phlebotomus perniciosus & 52 & 86.6 \\
& Sergentomia minuta & 7 & 11.6 \\
& Phlebotomus papatasi & 1 & 1.6 \\
& Phlebotomus neglectus & 0 & 0 \\
& Phlebotomus perniciosus & 93 & 60.38 \\
Rural & Sergentomia minuta & 54 & 35.06 \\
& Phlebotomus papatasi & 6 & 3.9 \\
& Phlebotomus neglectus & 1 & 0.64 \\
\hline
\end{tabular}

island should be considered not dangerous for people and dogs because its specificity to transmit Leishmania major. This parasite causes $\mathrm{CL}$ and is endemic in several African countries, and thus potentially imported by migrants; a risk of local transmission is however limited because of the absence on the island of the natural reservoir hosts (rodents of the Gerbillidae family). This study represents the first description of $P$. neglectus on the island, one of the four $L$. infantum vectors present in Italy. The significance of the presence of this sand fly species mainly described on the Adriatic coast of Italy and in Balkan area needs more evidences and studies. Our results show that $P$. pernicious the most competent vector for L. infantum, abundantly circulates both in the urban center and in the peripheral parts of the island. The high presence of this sand fly species amplifies the risk for human population, both resident and tourists. The number of cases acquired on Lampedusa is difficult to establish due to the transient tourist and refugee populations and the long incubation period of ZVL. Some tourists may develop the infection months or years after their trip on the island, with no many possibilities to distinguish this event from other potential insect-contact happened in other areas of Europe. Resident population has frequent business trips in Sicily that is considered endemic too. To complicate the risk analysis is that on the island is only present an emergency hospital, so people suffering from chronic diseases need to go to $\mathrm{Si}$ cily or to other Italian regions for medical care. A particular sanitary risk is represented by the very large proportion of migrants continuously present on the island. It is well known that due to the large number of landings they form an overpopulation that stays on the island for many weeks. Most of them arrive on the island during the summer, the period of maximum intensity of the vectors, and their large majority, first children and women, should be considered immune-depressed by a very long trip at limit of survival condition. What role could play the parasite Leishmania in these immune-imbalanced hosts should be better investigated, even considering the scarce information on their future life-condition all around the 
Europe. In it cannot be excluded their role as source of other Leishmania species, such as the anthroponotic cutaneous form due to L. tropica [15].

\section{Conclusion}

Our findings confirm the very high CanL seroprevalence on Lampedusa island. The high proportion of seropositive dogs and the presence of competent vectors demonstrate that $L$. infantum abundantly circulates in the island and may constitute a severe risk for people, living or hosting there. Sanitary measures should be considered by using large scale application of sand flies repellents and with the regular use of specific anti-Leishmania vaccine and drug treatment of sick dogs to limit their role of reservoir.

\section{Funding}

This study was funded by 'ISTITUTO ZOOPROFILATTICO SPERIMENTALE DELLA SICILIA", Palermo, Italy code number: IZS SI /15 RC.

\section{Availability of data and materials}

The dataset supporting the conclusions of this article is available at ISTITUTO ZOOPROFILATTICO SPERIMENTALE DELLA SICILIA", Via Gino Marinuzzi,3, 90129- Palermo, Italy.

Data supporting the conclusions of this article are included within the article in Tables 1 and 2. The datasets used and/or analyzed during the current study are available from the corresponding author on reasonable request.

\section{Authors' contributions}

VFM, MG, GO conceived the study; participated to clinical activities and wrote the manuscript. SS performed clinical examination and blood and lymph node samples; FV conceived the study; was the responsibility of all laboratory investigations; performed capture of sand flies. SM, performed statistical analysis. AT performed capture and identification of sand flies. All authors have read and approved the final manuscript.

\section{Ethics approval}

The study was evaluated by Animal Welfare Committee of University Federico II, Naples, Italy, reference number: 26719/2018. The collection of biological samples was performed in accordance with the national guidelines for animal welfare. A written consent for clinical procedures was obtained from the owners. The Animal Welfare Committee of University Federico II, approved this method of consent.

\section{Consent for publication}

Not applicable

\section{Competing interests}

The authors declare that they have no competing interests.

\section{Publisher's Note}

Springer Nature remains neutral with regard to jurisdictional claims in published maps and institutional affiliations.

\section{Author details}

'Department of Veterinary Medicine and Animal Production, University of Naples Federico II, 80137 Naples, Italy. ${ }^{2}$ Istituto Zooprofilattico Sperimentale della Sicilia - CreNaL, 90129 Palermo, Italy. ${ }^{3}$ DVM, Lampedusa, Italy.

Received: 16 March 2018 Accepted: 31 August 2018

Published online: 19 September 2018

\section{References}

1. Gradoni L. Epidemiological surveillance of leishmaniasis in the European Union: operational and research challenges. Euro Surveill. 2013;18(30):20539.

2. Moreno J, Alvar J. Canine leishmaniasis: epidemiological risk and the experimental model. Trends Parasitol. 2002;18(9):399.
3. Dujardin JC, Campino L, Cañavate C, Dedet JP, Gradoni L, Soteriadou K, et al. Spread of vector-borne diseases and neglect of Leishmaniasis. Europe Emerg Infect Dis. 2008;14(7):1013.

4. Franco AO, Davies CR, Mylne A, Dedet JP, Gállego M, Ballart C, et al. Predicting the distribution of canine leishmaniasis in western Europe based on environmental variables. Parasitology. 2011;138(14):1878.

5. Romagnoli P. Macr' G, Gradono L, maroli M. Serological survey on canine leishmaniasis and first record of phlebotomine sand flies in the southest Italian focus of leishmaniasis: Lampedusa island, Sicily. Parassitologia. 2002; 44:154.

6. Foglia Manzillo V, Di Muccio T, Cappiello S, Scalone A, Paparcone R, Fiorentino $\mathrm{E}$ et al. Prospective study on the incidence and progression of clinical signs in naiive dogs naturally infected by Leishmania infantum. PLoS Negl Trop Dis. 2013;7(5):e2225. https://doi.org/10.1371/journal.pntd.0002225. Print 2013.

7. Moreira MA, Luvizotto MC, Garcia JF, Corbett CE, Laurenti MD. Comparison of parasitological, immunological and molecular methods for the diagnosis of leishmaniasis in dogs with different clinical signs. Vet Parasitol. 2007; 145(3-4):245.

8. Office international des epizooties (O.I.E.). Manual of diagnostic tests and vaccines for terrestrial animals. 2014 2(1)11.

9. Reale S, Lupo T, Migliazzo A, Di Mauro C, Ciprì V, Calderone S, et al. Multilocus microsatellite polymorphism analysis to characterize Leishmania infantum strains isolated in Sicily. Transbound Emerg Dis. 2010;57(1-2):37.

10. Khalid N, Elnaiem D, Aboud M, Al Rabba F, Tripet F. Morphometric and molecular differentiation of Phlebotomus (Phlebotomus) sandflies. Med Vet Entomol. 2010;24(4):352.

11. Latrofa MS, Annoscia G, Dantas-Torres F, Traversa D, Otranto D. Towards a rapid molecular identification of the common phlebotomine sand flies in the Mediterranean region. Vet Parasitol. 2012;184(2-4):267.

12. Veterinary Epidemiology, Fourth Edition. $\odot 2018$ John Wiley \& Sons Ltd. Published 2018 by John Wiley \& Sons Ltd. Companion website: www.wiley. com/go/veterinaryepidemiology

13. Gramiccia M. Recent advances in leishmaniosis in pet animals: epidemiology, diagnostics and anti-vectorial prophylaxis. Vet Parasitol. 2011; 181(1):23.

14. Gramiccia M, Scalone A, Di Muccio T, Orsini S, Fiorentino E, Gradoni L. The burden of visceral leishmaniasis in Italy from 1982 to 2012: a retrospective analysis of the multi-annual epidemic that occurred from 1989 to 2009. Euro Surveill. 2013;18(29):20535.

15. Di Muccio T, Scalone A, Bruno A, Marangi M, Grande R, Armignacco O et al. Epidemiology of Imported Leishmaniasis in Italy: implications for a European Endemic Country. PLoS One. 2015;10(6):e0129418. https://doi.org/ 10.1371/journal.pone.0129418. eCollection 2015.
Ready to submit your research? Choose BMC and benefit from:
- fast, convenient online submission
- thorough peer review by experienced researchers in your field
- rapid publication on acceptance
- support for research data, including large and complex data types
- gold Open Access which fosters wider collaboration and increased citations
- maximum visibility for your research: over $100 \mathrm{M}$ website views per year
At $\mathrm{BMC}$, research is always in progress.
Learn more biomedcentral.com/submission 